\title{
Research on the Growth of Breast Tumor
}

\author{
Jinrui Huang
}

\author{
Indiana Area Senior High, Indiana PA, 15701, United States \\ Gmail: jrhuang1225@gmail.com
}

\begin{abstract}
Malignant breast tumor or cancer is notorious for its rampant spread over to any other parts of body and can be potentially a fatal disease. Many theoretical studies on the growth kinetics of breast tumor have been carried out in order to generalize the fundamental characteristic of it. This knowledge, in turn, can then be applied to actual clinical practice to give prediction as early as possible. The CoMPaS model is such a theoretical tool to predict when and how the tumor volume may develop. It handles the primary tumor and the secondary distant metastatic tumor simultaneously, and applies exponential as well as approximate linear dynamics to describe the growth of tumors even when they are still in the nonvisible stage, so it is helpful to meet the goal "find early, save early". In this paper, we make efforts to understand the details of CoMPaS model. The underlying equations are first introduced and derived, then one typical solution is given. The original paper seems to ignore the linear growth and keep only the exponential one, which contradicts the experiment fact, so we come up with a remedy assumption that the linear growth is switched on very lately, then our results match well those from the original paper. The issue should be raised here is when the linear growth comes into play.
\end{abstract}

Keywords: Breast tumor, CoMPas, Primary tumor (PT), Secondary distant metastatic tumor (MTS), Exponential/Linear model

\section{INTRODUCTION}

Breast tumor, alternatively known as breast cancer (BC), proliferates due to the abnormal growth of cells of breast in an uncontrollable manner. Women are more vulnerable to breast cancer because of lifelong exposure to estrogen. It is the main cause of cancer mortality in women and accounts for approximately $20-25 \%$ of all the cancer types in women [1]. The defining feature of $\mathrm{BC}$ is its rapid growth beyond breasts that results in invasion to other organs through blood and lymph vessels. When BC spreads to any other parts of the body, it is said to have metastasized [2]. This multiple proliferation of secondary distant metastatic tumor (MTS), even after the primary tumor (PT) is removed, accounts for most death from cancer.

For more than 60 years, tumor growth kinetics has been theoretically and experimentally investigated extensively [3] in analyzing the behavior of breast cells. This study is of immense biological significance since the early detection of the growth of the tumor may help the patient take the necessary treatment in time for their lives. From the view of theoretical simulation, finding algorithm to predict the growth of tumors have aroused the interests of cancer researchers since the very early days of BC research. Many studies have been carried out to understand the duration from the first $\mathrm{BC}$ cell appearance till the death of a patient, often referred to as the natural history of BC growth. These studies brought various mathematical models in to describe PT and MTS growth together or separately [4].

Mathematical descriptions [5] include the exponential-linear model where the tumor growth presumes all cells proliferate with constant cell cycle duration, hence simply leading to exponential growth case; the logistic and Gompertz model where relative tumor growth rate is decreased linearly with respect to time in order to incorporate the competition for nutrition or space between cancer cells and hence qualitatively reproduce the experimentally observed growth slowdown that is consistent with general patterns of organ and organismal growth [6]; as well as more complicated dynamic capacity model to reflect that the tumor vasculature is inhibited to the surface of tumor only [7]. Each model stresses a particular aspect of growth mechanism.

This paper is supposed to understand the CoMPaS model and its application. Based on the observed data, 
the growth of PT and the secondary distant MT S can be captured best, at least at the early stage of tumor, by an exponential description. This exponential growth is incorporated into the CoMPaS model, which can be optionally followed by a much slower linear growth, and this model even goes further since it handles PT and MTS together, not only describing the whole natural history of $\mathrm{BC}$, but predicting the period of clinical MTS manifestation after PT resection [8]. Next we brief this model. The details come from the reference [8].

\section{COMPAS MODEL}

The ComPaS is such a model that describes a twostage development of BC, initially an exponential growth then followed by an asymptotic linear growth when the tumor size is appreciable or even larger. The model consists of both determinate nonlinear and linear equations and is compliant to the TNM classification [9]. As mentioned above, secondary distant MTS may appear at various time in different organs, the correlation between PT and MTS in this model is purely along time line of development no matter how far they are separate from each other, and moreover, the early detection of tumor transfer is limited by a visible size, often specified by the tumor diameter $1-5 \mathrm{~mm}$.

The whole natural history of $\mathrm{BC}$ growth contains 60 doublings, that is, the proliferation finishes 60 cycles and is enough for the tumor to reach its threshold size (around $100 \mathrm{~mm}$ ) and kill the patient. This history may include the following four stages: the non-visible period of PT growth starting from the appearance of the first tumor cell until its growth can be detected; the visible period of PT growth referring to the duration from the point that it reached a visible size up to the pre-surgery size; the nonvisible period of MTS growth as the period from date of PT resection to a visible size for at least one MTS and the visible period of MTS growth as the period from diagnosis of a MTS to its achievement of the lethal size, the second and third of which are technically separated by the date of resection without a clear-cut definite way to distinguish.

The central descriptive variable of the CoMPaS model is the tumor volume, denoted by $\mathrm{V}$, being proportional to the total number of cells in the tumor. $\mathrm{V}$ varies as a function of time $t$. It assumes that PT and all MTSs share the same natural growth rate. All cells proliferate with the same constant DT, cell cycle duration time. As for the initial time of the appearance of the first metastatic cell of the secondary distant MTS, the model arbitrarily chooses to coincide with the 20th doubling of the primary $\mathrm{BC}$. It contains the following two deterministic equations,

$$
\frac{\mathrm{dV}}{\mathrm{dt}}=\frac{\ln 2}{\mathrm{DT}} \mathrm{V}, \mathrm{t} \leq \mathrm{DT} \log _{2}\left(\frac{\theta \mathrm{DT}}{\ln 2 \mathrm{~V}_{0}}\right)
$$

$$
\begin{gathered}
\frac{\mathrm{dV}}{\mathrm{dt}}=\theta \ln \mathrm{V}, \mathrm{t}>\mathrm{DT} \log _{2}\left(\frac{\theta \mathrm{DT}}{\ln 2 \mathrm{~V}_{0}}\right) \\
\mathrm{V}(\mathrm{t}=0)=\mathrm{V}_{0} \\
60=\mathrm{PT}_{\log }+\text { Nonvis }_{\log }+\mathrm{Vis}_{\log } \\
\mathrm{TVDT}_{\text {non }}=\mathrm{TVDT}_{\text {vis }}=\frac{\text { Nonvis }_{\text {days }}+\text { Vis }_{\text {days }}}{\text { Nonvis }_{\text {log }}+\text { Vis }_{\text {log }}}
\end{gathered}
$$

V0 - the initial fixed volume

DT - the average doubling time or the cycle time needed for a cancer cell to proliferate. It is expressed by the product of the total proliferative cells and the factor $\ln 2$ / DT. Using this factor converts time to the unit of number of doublings.

$\theta$ - the parameter to define the following linear phase

PTLog - the number of doublings for the growth period of PT

Nonvislog - the number of doublings for the nonvisible growth period of MTS

Vislog - the number of doublings for the visible growth period of MTS

TVDT -tumor volume doubling time

Clearly, the exponential growth is described by $\mathrm{V}=$ $\mathrm{V}_{0} \mathrm{e}^{\frac{\ln 2}{\mathrm{DT}} \mathrm{t}}$, then followed an approximate linear growth stage, $\operatorname{li}(\mathrm{V})=\theta t, \mathrm{l}(\mathrm{V})$ being the logarithmic integral function. It is defined as,

$$
\operatorname{li}(\mathrm{V})=\int_{0}^{\mathrm{V}} \frac{\mathrm{dt}}{\ln \mathrm{t}}
$$

In the first stage, the size of $\mathrm{V}$ is doubled to be $2 \mathrm{~V}$ after a cycle of growth. When $\mathrm{t}=\mathrm{DT} \log _{2}\left(\frac{\theta \mathrm{DT}}{\ln 2 \mathrm{~V}_{0}}\right)$,

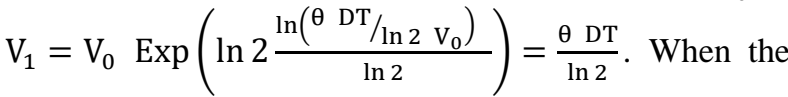
tumor volume is greater than this value, the linear growth will then be switched on and controlled by the second deferential equation. The time dependence of $\mathrm{V}$ at this stage can be solved from the inverse function of li(V), see the reference [10].

$$
\begin{aligned}
\operatorname{li}(\mathrm{V})-\operatorname{li}\left(\mathrm{V}_{1}\right)= & \theta(\mathrm{t}-\tau), \\
& \frac{\ln 2}{\theta \mathrm{DT}} \operatorname{li}(\mathrm{V})-\frac{\ln 2}{\theta \mathrm{DT}} \operatorname{li}\left(\mathrm{V}_{1}\right) \\
= & \frac{\ln 2}{\mathrm{DT}}(\mathrm{t}-\tau)
\end{aligned}
$$

Now inverse the function on both sides. As a reminder, if both axes are stretched by the same amount, the function relation remains the same, that is, $y=f(x)$, $\mathrm{f}^{-1}$ (ay) $=\mathrm{ax}$.

$$
\frac{\ln 2}{\theta \text { DT }} \mathrm{V}-\frac{\ln 2}{\theta \text { DT }} \mathrm{V}_{1}=\mathrm{li}^{-1}\left[\frac{\ln 2}{\mathrm{DT}}(\mathrm{t}-\tau)\right]
$$




$$
\frac{\mathrm{V}}{\mathrm{V}_{1}}=1+\mathrm{li}^{-1}\left[\frac{\ln 2}{\mathrm{DT}}(\mathrm{t}-\tau)\right]-1.45
$$

where $V_{1}$ is the tumor volume at the moment of stage transition. The root 1.45 is added in order to make $V_{1}=$ $\mathrm{V}(\tau)$ since $\operatorname{li}(0)=1.45$. The graph below illustrates the dynamics of two stages. The graph on the left shows how $\operatorname{li}(\mathrm{V})$ changes with tumor volume $\mathrm{V}$, and its inverse function, shown on the right panel, could describe how $\mathrm{V}$ increases with time for the second stage. Actually, only the curve far to the red spot $\left(1.45 \mathrm{~V}_{0}\right)$ is used to describe the growth dynamics. After many cycles, linearity seems to be a good approximation for large value of time. As a comparison, the inset gives a glimpse of exponential growth that changes much more dramatically in the early stage. The transition time between two dynamics should be large enough so that the tumor growth can be well described linearly with time. That means the size of BC tumor must have been very large when the linear dynamics is turned on.
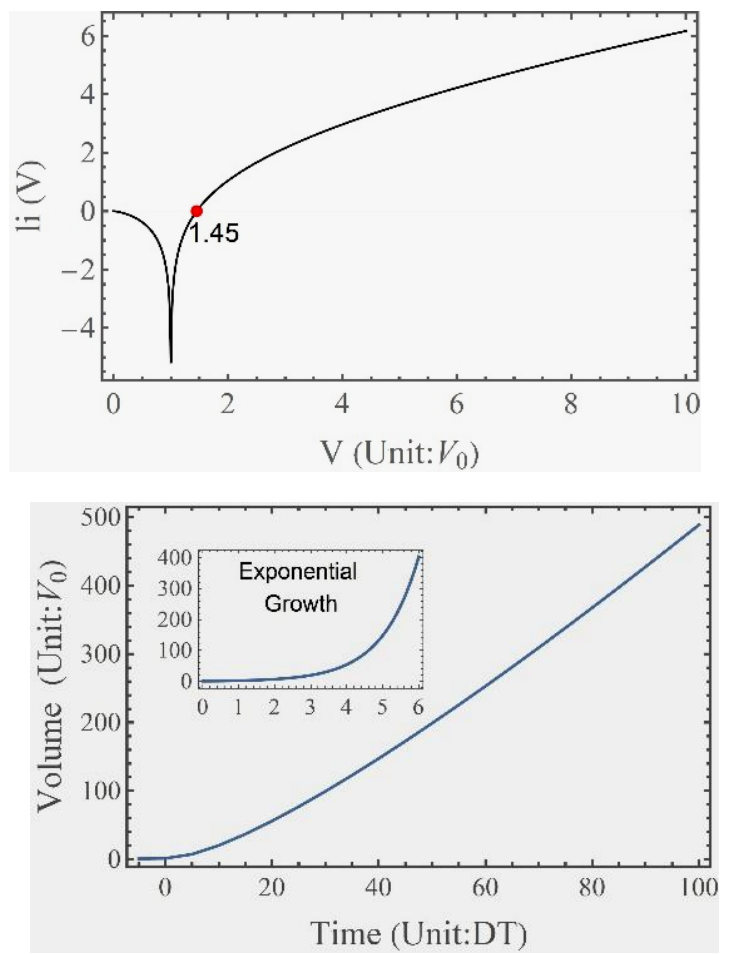

Figure 1. The appearance of linear stage with exponential stage as a comparison

Now we derive the important prediction by the CoMPaS model. In the following description, the number of cells $\mathrm{N}$ is essential a synonym of the word "size", more directly, the diameter of the tumor. The diameter of the very first tumor cell equals $10 \mu \mathrm{m}$. After the 10th doubling, the diameter equals $100 \mu \mathrm{m}$, equivalent to $\mathrm{N}=10^{3}$. After the 20th doubling, the diameter of tumor equals $1 \mathrm{~mm}\left(\mathrm{~N}=10^{6}\right)$. And it continues to increase to reach $10 \mathrm{~mm}$ with 30 doublings, $\mathrm{N}=10^{9}$. Then it needs 40 doublings for the tumor size to reach $100 \mathrm{~mm}$ $\left(\mathrm{N}=10^{12}\right)$. The diameter of a tumor of more than $10 \mathrm{~cm}$ is often taken as the lethal size threshold of that tumor.

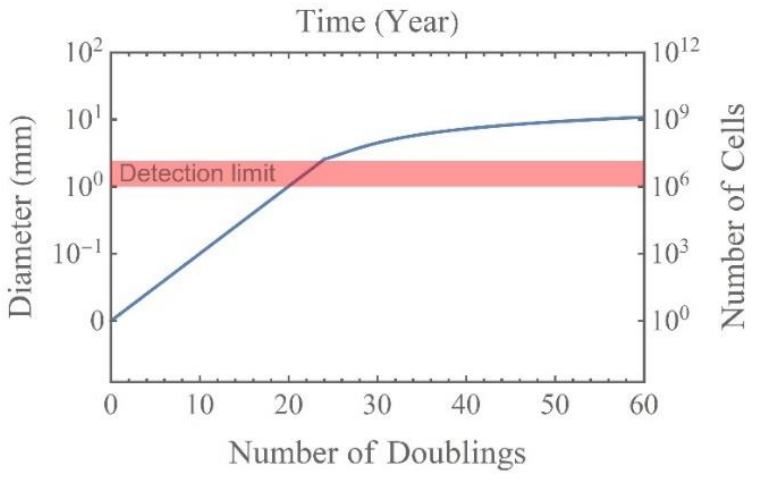

Figure 2. The CoMPaS model predicts a two-stage of $\mathrm{BC}$ growth

The above graph uses this size scale and has a test on when the second stage comes into play. If the second stage started as soon as the tumor can be detected, the 10 $\mathrm{cm}$ size could have been put off to a late moment beyond 60 doublings, which leads to a much longer unrealistic life expectation (longer than 60 doublings).

This paper mainly follows the thread of TN's work. They repeated the calculation for each of four stages. All of the results shared the same appearance, that is, only exponential growth (straight line in logarithmic graphs) were drawn, so they did not take into account the influence of the approximate linear growth, as a result, the whole natural growth history of $\mathrm{BC}$ could terminate within 60 doublings. That is roughly equivalent to 11 years life span. Nevertheless, this trend seems to contradict the experiment fact that the relative growth rates of tumor cells decrease with time [5]. As a compromise, we assume that the linear stage will be switched on only after 40 50 doublings so that the main conclusion of TN's work that the whole growth history is dominated solely by the exponential growth could still stand, and leave the door to allow the linear growth description and adjust life span later. With this assumption, the results in TN's paper are reproduced well here.

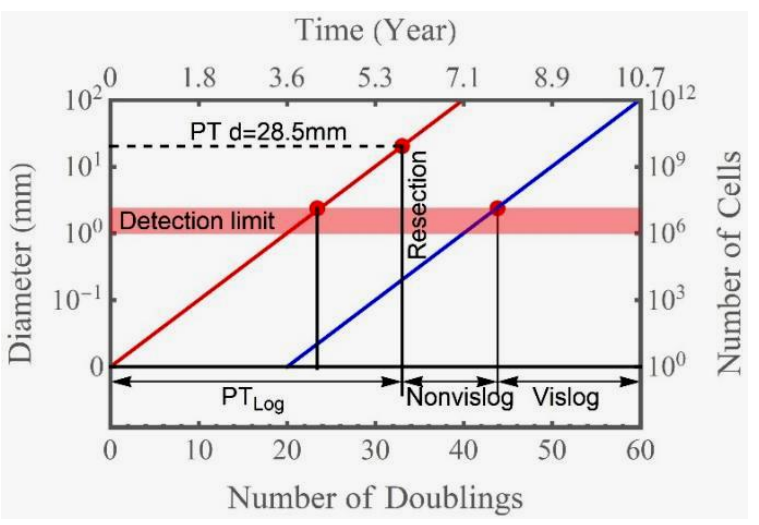

Figure 3. The result of TN's paper is reproduced well.

TN's paper uses the nomenclature of $\mathrm{BC}$ classification. The tumor are said to be in different stages 
according to their sizes of PT. That is where the symbols T1N0M0, T2N0M0 and T3N0M0 come from. In the early stage, $\mathrm{BC}$ can even be divided into sub-level stages such as T1aN0M0, T1bN0M0 and so on. Here N0 represents no lymph nodes appearance yet, and $\mathrm{M}$ whether or not the secondary distant MTS has appeared and evolved. When you look through the figures $1 \sim 8$, you will find they essentially give the same result, so we can simply choose randomly any one of them to do a calculation. Take T2N0M0 as an example, the diameter of this stage lies in the range $20 \leq \mathrm{d} \leq 50 \mathrm{~mm}$. Our result is shown in the above log plot. The same calculation can certainly be done for any other stages.

In the calculation, DT is taken as 65 70 days from their paper. Clinically, with only two measurements $\left(t_{1}, V_{1}\right)$ and $\left(t_{2}, V_{2}\right)$ of the PT sizes, DT can be calculated from the following [11], DT $=\frac{\Delta \mathrm{t} \ln 2}{\ln V_{2}-\ln V_{1}}$, and time in years is obtained from the number of doublings. As mentioned before, the first metastatic cell appears at the 20th doubling of PT. It is natural to assume that the growth rate of MTS is equal to that of PT, so two lines with same slope are expected. An advantage of the model is it can provide the information on nonvisible growth period for both PT and MTS. You can easily read both from the above graph.

\section{CONCLUSION}

The CoMPaS is a model to describe how the $\mathrm{BC}$ evolves with time, including an exponential growth and an optionally slower asymptotic linear growth. It consists of deterministic equations and can find itself to be consistent with the TNM classification. The main feature of this model is it is able to describe both the PT growth and secondary distant MTS growth as a whole, and allow us to calculate the number of proliferation doublings for all the four growth stages including nonvisible PT, visible PT, nonvisible MTS and visible MTS periods. A mathematical modelling on non-visible growth period helps to detect the appearance period of the first tumor cell and the first metastatic cell in different organs so that patients with BC may start treatment at the early stages and increase survival rate.

TN's work [8] illustrates how this model works. They seem to pay attention only to the exponential growth stage and does not include a slower linear growth of BC, but they pointed out that this model has been validated using 10-year and 15-year survival clinical data considering tumor stage and PT diameter, which seems to lend the ground to exclude the linear growth or justify our assumption of late time switch-on for that stage.

The CoMPaS model does not necessitates any costly diagnostic tests and can be the first predictor to make forecasts using only current patient data without the need of additional statistical data. It is able to build the whole natural history of the $\mathrm{BC}$ stages and predict the survival rate. They can be easily read out from the resulting graph as shown in Fig 3. To compare forecasts with real data and estimate the quality of the CoMPaS model, this model can be linked to a database [6] to test the model precision. Data can also be accumulated for future model improvement.

\section{REFERENCES}

[1] Shah R, Rosso K, Nathanson SD. Pathogenesis, prevention, diagnosis and treatment of breast cancer. World J Clin Oncol 2014; 5: 283-298. https://doi.org/10.5306/wjco.v5.i3.283 PMID: 25114845.

[2] "What Is Breast Cancer?" Centers for Disease Control and Prevention, 22 Sept. 2021, cdc.gov/cancer/breast/basic_info/what-is-breastcancer.htm.

[3] Steel GG (1977) Growth kinetics of tumors. Clarendon Press. Oxford.

[4] Rodriguez-Brenes IA, Komarova NL, Wodarz D. Tumor growth dynamics: insights into evolutionary processes. Trends Ecol Evol 2013; 28: 597-604. https://doi.org/10.1016/j.tree.2013.05.020 PMID: 23816268.

[5] Benzekry S, Lamont C, Beheshti A, Tracz A, Ebos JML, Hlatky $\mathrm{L}$ and Hahnfeldt $\mathrm{P}$ Classical Mathematical Models for Description and Prediction of Experimental Tumor Growth PLoS Comput Biol 10(8): e1003800. doi:10.1371/journal.pcbi.1003800.

[6] Spratt JA, von Fournier D, Spratt JS, Weber EE. Decelerating growth and human breast cancer. Cancer 1993; 71: 2013-2019. PMID: 8443753.

[7] Ribba B, Watkin E, Tod M, Girard P, Grenier E, et al. (2011) A model of vascular tumour growth in mice combining longitudinal tumour size data with histological biomarkers. Eur J Cancer 47: 479-490. Available: http://www. ncbi.nlm.nih.gov/pubmed/21074409.

[8] Tyuryumina EY and Neznanov AA Consolidated mathematical growth model of the primary tumor and secondary distant metastases of breast cancer (CoMPaS). PLoS ONE 13(7): e0200148. https://doi.org/10.1371/journal. pone.0200148.

[9] Clare SE, Nakhlis F, Panetta JC. Molecular biology of breast cancer metastasis. The use of mathematical models to determine relapse and to predict response to chemo-therapy in breast cancer. Breast Cancer Res 2000; 2: 430-435. pmid:11250737.

[10]https://mathematica.stackexchange.com/questions/6 7319/inverse-of-logintegral 
[11]https://www.researchgate.net/publication/32559351 3_THE_INVERSE_OF_THE_LOGARITHMIC_I NTEGRAL.

[12] Schwartz M. A biomathematical approach to clinical tumor growth. Cancer 1961; 14: 12721294. PMID: 13909709. 